\title{
Statistical mechanics of double-helical polymers
}

\author{
Alvise De $\mathrm{Col}^{1,2}$ and Tanniemola B. Liverpool ${ }^{1}$ \\ ${ }^{1}$ Condensed Matter Theory Group, Blackett Laboratory, Imperial College, London SW7 2BZ \\ ${ }^{2}$ Theoreticshe Physik, ETH Hönggerberg, CH-8093 Zürich, Switzerland
}

(November 20, 2018)

\begin{abstract}
We introduce a simple geometric model for a double-stranded and double-helical polymer. We study the statistical mechanics of such polymers using both analytical techniques and simulation. Our model has a single energy-scale which determines both the bending and twisting rigidity of the polymer. The helix melts at a particular temperature $T_{c}$ below which the chain has a helical structure and above which this structure is disordered. Under extension we find that for small forces, the behaviour is very similar to worm-like chain behaviour but becomes very different at higher forces.
\end{abstract}

Recent developments in single molecule manipulation techniques has led to the detailed study of the mechanical properties of DNA and other biomolecules as well as their response to applied fields. The model most used in the study of the large-scale properties of biopolymers is that of the worm-like chain [1]] in which the polymer flexibility (structure) is determined by a single length, the persistence length $L_{p}$ which measures the tangent-tangent correlations. For example, DNA has a persistence length $L_{p} \approx 50 \mathrm{~nm}$. Such coarse-grained models are needed to understand the statistical behaviour of long chains with a large number of monomers. They are complementary to chemically specific models which describe accurately the local effects of external fields but generally cannot deal with long chains [2]. Generalisations of the worm-like chain to introduce twist degrees of freedom have also been proposed [3]. Whilst the worm-like chain model and its generalisations give a good account of DNA under small fields (perturbations), it fails when these perturbations become large, e.g. under tensional forces above $65 \mathrm{pN}$ [4,5]. Such situations are biologically relevant in for example in DNA replication and repair.

DNA is double-stranded and helical. We introduce a geometric model which includes three important ingredients of DNA in B-form. First, the double-stranded nature of DNA given by the two phosphate backbones; second, the hydrogen bonds that keep the two strands together and third, the interactions between the bases that bring about the twisted structure. Similar models could be used to study other double-helical polymers like F-actin. The model is a generalisation of the double-stranded semiflexible (ribbon) polymer introduced by Liverpool, Golestanian and Kremer (LGK) [6] which takes into account the first two aspects but ignores the third. Other ribbon models have also been proposed by a number of authors [7]. It will be our conclusion that it is exactly this third property, the base-stacking interaction, which can account for many of the elastic properties of DNA. Unlike Z. Haijun et al [8], who proposed another generalisation of the LGK model with base-stacking interactions, we find that the rigidity of the individual strands is irrelevant for the effective persistence length, $L_{p}$. This is consistent with the large difference be- tween the persistence length of single and double-stranded DNA measured experimentally. Our model can easily be extended to include excluded volume and electrostatic interactions which for the moment have been ignored [9]. We do not attempt to make quantitative comparison with experiments but point out qualitative differences between simpler models. Our aim is rather to suggest a minimal model required to understand experimental results.

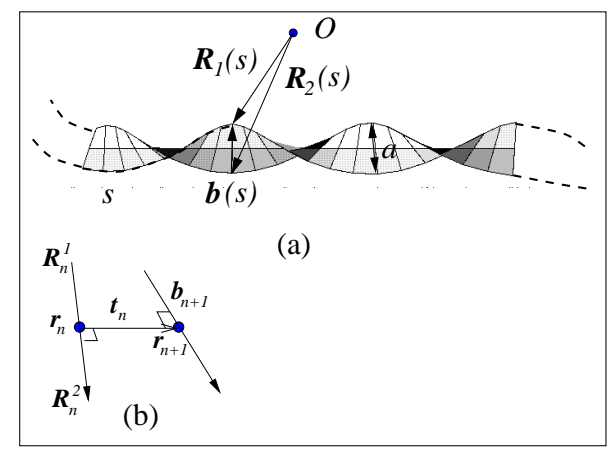

FIG. 1. (a) The schematic double-helical polymer with $\mathbf{R}_{2}(s)=\mathbf{R}_{1}(s)+a \mathbf{b}(s)$. The mid section (solid) is in the minimum energy configuration. (b) The discretisation of the double-helix used in the simulation.

The polymer is embedded in three dimensional space. As for the ribbon, we see qualitatively different behaviour for the high and low temperature regimes with a melting of the helix at a particular temperature $T_{c} \simeq$ $\left(\frac{4}{9}\right)^{2}\left[\frac{5+(2 P / \pi a)^{2}}{1+(2 P / \pi a)^{2}}\right]^{3 / 2} B / k_{B} a$ where $P$ and $a$ are respectively the pitch and diameter of the helix at $T=0$ and $B$ the 'stiffness' of the base-stacking interaction. Below $T_{c}$, there is a helical structure of the chain whilst above $T_{c}$, the helical structure vanishes. Unlike the ribbon, both the bend and twist rigidity of the double-helical polymer are determined by the basestacking interaction and the stiffness of the individual strands is irrelevant. We also study the response of the double-helical polymer to an extensional force and find that for small forces, the mechanical response is very similar to that of a worm-like chain but becomes significantly different at higher forces. 
The system is composed of two semi-flexible chains modelling the sugar phosphate backbones, each with rigidity $A$, whose embeddings in 3-dimensional space are defined by $\mathbf{R}_{1}(s)$ and $\mathbf{R}_{2}(s)$. Because of the covalent bonds $\left(E_{C b} \sim\right.$ $100 k_{B} T$ at room temperature) between the alternating phosphate and sugar molecules, the backbones are effectively inextensible at the forces and temperatures we consider. Similarly, the H-bonds $\left(E_{H b} \sim 10 k_{B} T\right.$ ) between the two backbones are assumed to keep the distance between the chains constant.

The separation of the strands is fixed and given by $a$, and the H-bonds give the constraint $\mathbf{R}_{2}(s)=\mathbf{R}_{1}(s)+a \mathbf{b}(s)$ where $\mathbf{b}(s)$ is a unit vector which we call the bond-orientation vector (see Fig. 1). The bond-orientation vector is perpendicular to both strands. The infinitesimal distance in 3-dimensional space between neighbouring points on a single strand is a function of the local curvature, $\partial_{s}^{2} \mathbf{R}_{i}(s)$ of the strands, where $\partial_{x} A(x) \equiv \partial A / \partial x$. The base-stacking interaction can be modelled by a potential $V_{i}\left(\partial_{s}^{2} \mathbf{R}_{i}, \mathbf{b}\right)$, where the subscript $i \in\{1,2\}$ refers to strand 1 or 2 , which is a function of the curvatures of the chains and the bond-vector only, with a minimum given by a symmetric double-helix. The Hamiltonian of the system is given by

$$
\begin{array}{r}
\mathcal{H}=\int d s\left\{V_{1}\left(\partial_{s}^{2} \mathbf{R}_{1}, \mathbf{b}\right)+V_{2}\left(\partial_{s}^{2} \mathbf{R}_{2}, \mathbf{b}\right)\right. \\
\left.+A\left(\left|\partial_{s}^{2} \mathbf{R}_{1}\right|^{2}+\left|\partial_{s}^{2} \mathbf{R}_{2}\right|^{2}\right)\right\} .
\end{array}
$$

The simplest example of such a potential which we will use for the remainder of this letter is the quadratic $V_{1}\left(\partial_{s}^{2} \mathbf{R}_{1}, \mathbf{b}\right)=$ $B\left(\partial_{s}^{2} \mathbf{R}_{1}-H \mathbf{b}\right)^{2}$ and $V_{2}\left(\partial_{s}^{2} \mathbf{R}_{2}, \mathbf{b}\right)=B\left(\partial_{s}^{2} \mathbf{R}_{2}+H \mathbf{b}\right)^{2}$. This is a potential of stiffness $B$ with a minimum when equivalent points on the two strands have equal and opposite curvatures, $\pm H$. More complicated potentials with several minima are also possible (see later). We note that we have not included the asymmetry of the DNA helix but a similar but more complicated model could be defined for an asymmetric doublehelix [9]. We implement the model by introducing the "midcurve" $\mathbf{r}(s): \mathbf{R}_{1}(s)=\mathbf{r}(s)+\frac{a}{2} \mathbf{b}, \mathbf{R}_{2}(s)=\mathbf{r}(s)-\frac{a}{2} \mathbf{b}$. In terms of the tangent to the mid-curve $\mathbf{t}(s)=\partial_{s} \mathbf{r}$ and the bond-director $\mathbf{b}$ the Hamiltonian of the system can now be written as

$$
\begin{aligned}
& \mathcal{H}[\mathbf{t}, \mathbf{b}]=\frac{B}{2} \int \mathrm{d} s \\
&\left.+\left(\partial_{s} \mathbf{t}(s)-\frac{a}{2} \partial_{s}^{2} \mathbf{b}(s)+H \mathbf{b}\right)^{2}\right],
\end{aligned}
$$

subject to the exact (local) constraints

$$
\left(\mathbf{t} \pm \frac{a}{2} \partial_{s} \mathbf{b}\right)^{2}=1, \quad \mathbf{b}^{2}=1, \quad\left(\mathbf{t} \pm \frac{a}{2} \partial_{s} \mathbf{b}\right) \cdot \mathbf{b}=0
$$

corresponding to the chain inextensibility, fixed distance between strands and definition of the bond vector respectively. We make the assumption that $B \gg A$ since the bending rigidity of single-stranded DNA is very small, $\left(A / k_{B} T<10 \AA\right)$, compared to the double-stranded form. We can define a typical length $\ell=B / k_{B} T$, which together with the strand separation $a$ and equilibrium radius of curvature $H^{-1 / 2}$ form the three relevant lengths of the problem. This completes the formulation of the model.

$T=0$ behaviour We can calculate the ground state of this system from the condition $\mathcal{H}=0$ which gives, $\mathbf{R}_{1}(s)=\frac{a}{2} \cos (\Theta s) \hat{e}_{1}+\frac{a}{2} \sin (\Theta s) \hat{e}_{2}+s \sqrt{1-\frac{a^{2} \Theta^{2}}{4}} \hat{e}_{3}$ and $\mathbf{R}_{2}(s)=-\frac{a}{2} \cos (\Theta s) \hat{e}_{1}-\frac{a}{2} \sin (\Theta s) \hat{e}_{2}+s \sqrt{1-\frac{a^{2} \Theta^{2}}{4}} \hat{e}_{3}$ where $\Theta=\sqrt{\frac{2 H}{a}}$. This is a double helix with a pitch: $P=\frac{2 \pi}{\Theta} \sqrt{1-\frac{a^{2} \Theta^{2}}{4}}$ and the bond-director field, $\mathbf{b}(s)=$ $-\cos (\Theta s) \hat{e}_{1}-\sin (\Theta s) \hat{e}_{2}$ where $\left\{\hat{e}_{i}\right\}$ are an orthonormal set of unit vectors.

Finite $T$ behaviour Inspite of the simplification of the model from using constraints, an exact analytic expression for the partition function of the model is still not available. For our analytic calculations, we use a very useful approximation [10] which describes correctly the equilibrium behaviour of correlation functions and even distribution functions, if the end effects are taken into account carefully [11]. This is a mean field approach that relaxes the local constraints to global ones [10,11]. It may be thought of as a self-consistent theory and corresponds to the saddlepoint evaluation of path integrals over the lagrange multipliers [12]. This approach fails for some dynamical properties [13] which are outside the scope of this letter. To implement this, we add a variational term to our Hamiltonian $\frac{\mathcal{H}_{m}}{k_{B} T}=\int \mathrm{d} s\left[\frac{b}{\ell}\left(\mathbf{t}-\frac{a}{2} \partial_{s} \mathbf{b}\right)^{2}+\frac{b}{\ell}\left(\mathbf{t}+\frac{a}{2} \partial_{s} \mathbf{b}\right)^{2}+\frac{c a^{2}}{4 \ell^{3}} \mathbf{b}^{2}\right.$ $\left.+\frac{e}{\ell}\left(\mathbf{t}-\frac{a}{2} \partial_{s} \mathbf{b}\right) \cdot \mathbf{b}+\frac{e}{\ell}\left(\mathbf{t}+\frac{a}{2} \partial_{s} \mathbf{b}\right) \cdot \mathbf{b}\right]$, where $b, c$, and $e$ are dimensionless constant Lagrange multipliers .

Two useful geometric parameters $u=H a / 2$ and $v=$ $4(\ell / a)^{2}$ can be used to characterise the double-helical polymer. Note that $v$ is proportional to $T^{-2}$ and can be viewed as a measure of temperature. We then determine the constants self consistently by demanding the constraints of Eq.(3) to hold on average, where the thermal average is calculated by using the total Hamiltonian $\mathcal{H}+\mathcal{H}_{m}$. The self-consistency lead to the following set of equations for the constants $b$ and $c:\left(c+u^{2} v^{2}\right)\left[(b-u v)+\sqrt{c+u^{2} v^{2}}\right]=\frac{9 v^{2}}{32} ; \frac{1}{4 \sqrt{2 b}}+$ $\frac{1}{3} \frac{1}{v} \sqrt{c+u^{2} v^{2}}=\frac{1}{3}$ and $e=0$. The above equations, which are nonlinear and difficult to solve exactly, determine the behaviour of $b$ and $c$ functions of $u$ and $v$. One can solve the equations analytically in some limiting cases corresponding to $T \rightarrow 0$ and $T \rightarrow \infty$. For $v \rightarrow \infty(T \rightarrow 0)$ and $H \neq 0$ we find, $b \sim \frac{9}{32} \frac{1}{(1-u)^{2}}, c \sim \frac{9}{16}\left[\frac{1}{u^{2}}-\frac{1}{(1-u)^{2}}\right] u v$ and for $v \rightarrow 0$ $(T \rightarrow \infty)$ and $H \neq 0$, we obtain $b \sim \frac{9}{8}, c \sim\left(\frac{1}{4 u^{2}}-1\right) u^{2} v^{2}$ A full solution requires a numerical treatment and shows simple monotonic behaviour for both $c$ and $b$ [15].

We can then calculate the correlation functions. For the tangent-tangent correlation one obtains

$$
\langle\mathbf{t}(s) \cdot \mathbf{t}(0)\rangle=\frac{3}{4 \sqrt{2 b}} e^{-\sqrt{2 b} \frac{s}{\ell}} \stackrel{T \rightarrow 0}{\approx}(1-u) e^{-\frac{3}{4(1-u)} \frac{s}{\ell}}
$$

whereas for the bond-director field one obtains 


$$
\langle\mathbf{b}(s) \cdot \mathbf{b}(0)\rangle=\frac{3 i}{2 a^{2}} \frac{\ell^{2}}{\ell^{2} \sqrt{D}}\left[\frac{e^{i \sqrt{(u v-b)+\sqrt{D} \ell^{2}} \frac{s}{\ell}}}{\sqrt{(u v-b)+\sqrt{D} \ell^{2}}}+\frac{e^{-i \sqrt{(u v-b)-\sqrt{D} \ell^{2}} \frac{s}{\ell}}}{\sqrt{(u v-b)-\sqrt{D} \ell^{2}}}\right] \stackrel{T \rightarrow 0}{\approx} e^{-\frac{3}{8 u} \frac{s}{\ell}} \cos \left(\sqrt{\frac{2 H}{a} s}\right)
$$

We have defined a discriminant $D(u, v)=$ $\frac{1}{\ell^{2}} \sqrt{(b-u v)^{2}-\left(c+u^{2} v^{2}\right)}$. The tangent-tangent correlation (Eq.(4)) of the double-helical polymers is similar to that of a worm-like chain but with a length rescaling factor, and decays exponentially at all temperatures. Eq.(5) on the other hand, indicates a change of behaviour at $D=0$ i.e. $(b-u v)^{2}=c+u^{2} v^{2}$ for the bond-director correlation. The correlation is over-damped for $(b-u v)^{2}>c+u^{2} v^{2}$ (high temperatures), while it is under-damped (oscillatory) for $(b-u v)^{2}<c+u^{2} v^{2}$ (low temperatures). The interesting point $(b-u v)^{2}=c+u^{2} v^{2}$ happens for $v_{c} \simeq 9\left(\frac{9}{4(1+4 u)}\right)^{3}$, that leads to the value for $T_{c}$ quoted above. We also find a divergence in the specific heat, $C_{V}$ at $T_{c}$. We emphasise that it is not a thermodynamic phase transition in the sense of long-range ordering and broken symmetry. It is a cross-over that appears due to competing effects, and the transition is from a state with some short-range order to a state with a different short-range order. The cross-over (transition) point corresponds to a type of 'Lifshitz point' for a 1-d system [14]. At high temperatures, in addition to the effects we describe we also expect the H-bonds between the bases to break, leading to denaturation which we cannot treat in this model. In the low $T$ regime, we can read off the bend (tangent) persistence length $L_{T P}=\frac{4}{3} \ell(1-H a / 2)^{-1}$ and twist (bond) persistence length, $L_{B P}=\frac{4}{3} \ell H a$ and helical frequency $(2 H / a)^{1 / 2}$. Both the bend and twist rigidity of the double-helical polymer are given by the same energy scale $B=k_{B} T \ell$ and have little to do with the stiffness of the individual strands.

Force-extension: unwinding the helix Under a stretching force, which without loss of generality we orient along the z-axis $\mathbf{F}=F \hat{\mathbf{z}}$, the Hamiltonian becomes $\mathcal{H}_{F}=\mathcal{H}-\mathbf{F}$. $\int d s \partial_{s} \mathbf{r}$. Now $\left\langle t_{z}\right\rangle=\langle\mathbf{t} \cdot \hat{\mathbf{z}}\rangle \neq 0$ and we must use connected correlation functions to calculate the self-consistent equations. Defining $f=F a / 8$ we obtain the equations

$$
\left\{\begin{array}{l}
\frac{1}{4 \sqrt{2 b}}+\frac{1}{3}\left(\frac{f}{b}\right)^{2} v+\frac{1}{3} \frac{1}{v} \sqrt{c+u^{2} v^{2}}=\frac{1}{3} \\
\left(c+u^{2} v^{2}\right)\left[(b-u v)+\sqrt{c+u^{2} v^{2}}\right]=\frac{9 v^{2}}{32}
\end{array}\right.
$$

We can solve eqns. (6) for $b(u, v, f)$ and $c(u, v, f)$ and the extension per unit length $z(u, v, f)$ is simply:

$$
z=\frac{1}{L} \int_{0}^{L}\langle t(s)\rangle d s=\frac{f}{b} \sqrt{v}
$$

We can therefore obtain the force-extension relation for the double-helical polymer. Eq. 6 has been solved numerically and in Fig. I 2 we have plotted $z$ against $f$. In the inset we compare the force-extension curve to that of the WLC and find they are identical for small forces. We note the interesting fact that the mean field model can also be used to calculate the force-extension behaviour of the WLC. It has the simple compact form $f L_{p}=\frac{3 z}{2\left(1-z^{2}\right)^{2}}$. We find that this simple expression has an error of less than $4 \%$ compared to the 'exact' value of Marko and Siggia [11].

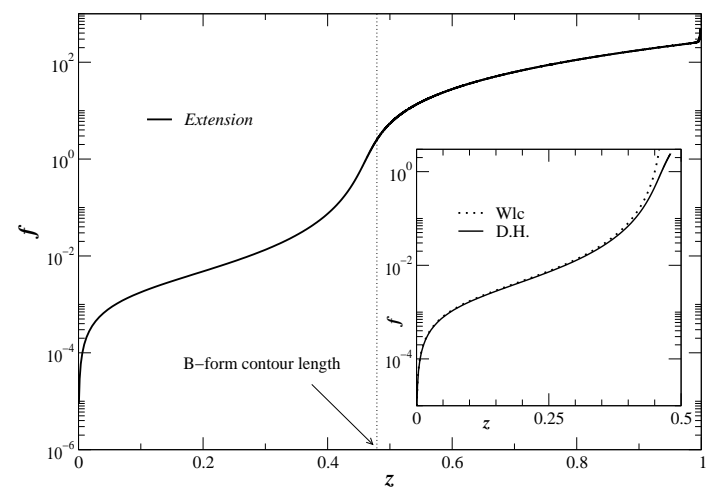

FIG. 2. Force-extension relation for the double-helical polymer (DHP). This is a log plot with $u=0.77$ and $v=115000$, typical values for B-DNA which corresponds to a low temperature $T<T_{c}$ (Note $f=F a / 8$ ). The dashed line shows the $T=0$ contour length $\sqrt{1-a^{2} \Theta^{2} / 4}$. Inset: comparison with worm-like chain at small forces.

Simulations We also performed Monte Carlo simulations of the double-helical polymers. We used a square lattice of size $\Delta$ to discretise the 'helical ribbon' and to implement all the constraints (see Fig. 11). The discretised Hamiltonian is given by

$$
\begin{aligned}
\beta \mathcal{H}=\ell \sum_{n=1}^{N-1} & \left(\frac{2}{\Delta}+H^{2} \Delta-\sum_{j=1}^{2}\left[\frac{\mathbf{t}_{n+1}^{j} \cdot \mathbf{t}_{n}^{j}}{\Delta}\right.\right. \\
& \left.\left.+(-1)^{j} H \mathbf{b}_{n} \cdot\left(\mathbf{t}_{n+1}^{j}-\mathbf{t}_{n}^{j}\right)\right]\right),
\end{aligned}
$$

where $n$ is the monomer label (position along the chain) and $j \in\{1,2\}$ the strand label (see Fig. 1). Since the Hamiltonian is local, we grew chains with a local algorithm. In our simulation the number of monomers $N$ was taken to be 1000 for each chain, $\Delta=1$ and $a=3$. Typical conformations at high and low temperatures are shown in Fig. 7 .

Typical bond correlation functions are plotted in Fig. B. From the bond and tangent correlation functions, we can obtain the bond and tangent persistence lengths. We calculate the bond correlation length as $L_{B P}=C_{B} u \ell$ and the tangent correlation length as $L_{T P}=C_{T} \ell$ with the constants given $C_{B}=1.09 \pm 0.09$ and $C_{T}=2.46 \pm 0.73$ respectively. A comparison with the mean field results shows that the results are the same to within a constant. This difference can be considered as due to finite corrections to the mean field solution due to higher order terms in the $1 / d$ expansion. 


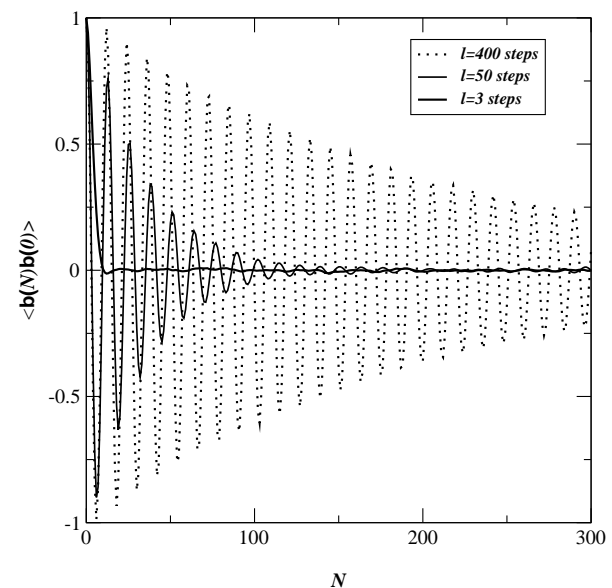

FIG. 3. The $\langle\mathbf{b}(s) \cdot \mathbf{b}(0)\rangle$ correlation function measured in the simulations for temperatures $\ell=400,50,3$ and $u=0.6$ corresponding to $T<T_{c}$ and $T>T_{c}$. The averages were done over 200 statistically independent samples.

In conclusion, we have studied the properties of a welldefined model of a double-helical, double-stranded semiflexible polymer using a mean-field analytical approach as well as extensive MC simulations. We have shown novel nontrivial differences between the high, low and zero temperature behaviour. A detailed comparison with the simulations and the mean-field solution shows qualitative and almost quantitative agreement.

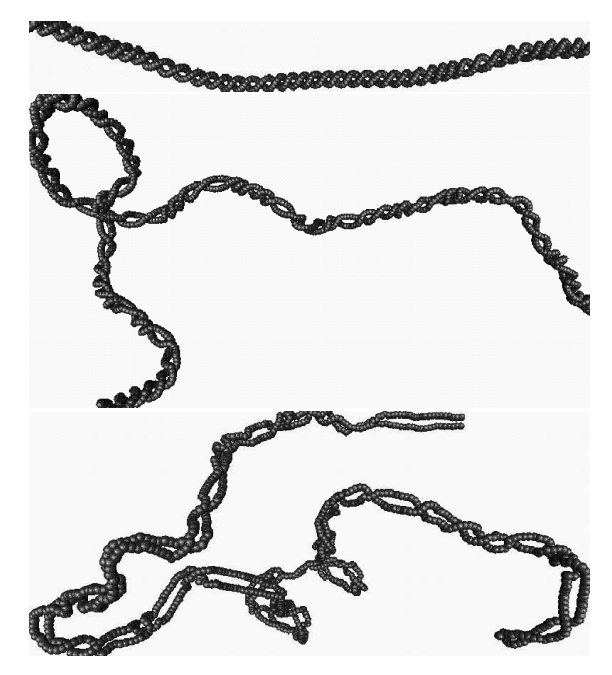

FIG. 4. Typical conformations at (a) low, (b) intermediate and (b) high temperatures

The fine structure studied in this model gives a different behaviour compared to a WLC. We see this most clearly when the double-helical polymer is subject to an external pulling force. It shows two distinct regimes. For low forces the dynamical response is simular to a WLC, with the most relevant energetic contribution given by the bending rigidity. At higher forces the extension changes abruptly and increases steeply as the double helical structure is unwound. This qualitatively agrees with the experimental results on over-stretched DNA [4,5], which nevertheless have a sharper transition and a flatter plateau. The graph is plotted on a log scale, so as to make clearer the complex behaviour of the force-extension curve. There are a two obvious ways in which a sharper transition could be obtained. First, we have used a simple quadratic potential for the base-stacking interaction corresponding only to an expansion about the minimum energy conformation. A more realistic potential with a cut-off and/or several minima could change the sharpness of and number of transitions. In addition one could imagine as in mechanical unzipping experiments on DNA, that there could be cooperative effects due to the effects of sequence disorder.

We have benefited from discussions with R. Everaers, R. Golestanian and K. Kremer.

[1] O. Kratky and G. Porod, Rec. Trav. Chim., 68, 1106 (1949) .

[2] W. K. Olson and V. B. Zhurkin, Curr. Opin. Struc. Bio., 10, 286 (2000).

[3] J.F. Marko and E.D. Siggia, Macromolecules, 27, 981 (1994); C. Bouchiat and M. Mezard, Phys. Rev. Lett. 80, 1556 (1998); S. Panyukov and Y. Rabin, Phys. Rev. Lett. 85, 2404 (2000) .

[4] S. B. Smith, Y. Cui, and C. Bustamante, Science 271, 795 (1996).

[5] P. Cluzel et al., Science 271, 792 (1996).

[6] T. B. Liverpool, R. Golestanian, and K. Kremer, Phys. Rev. Lett. 80, 405 (1998); T.B. Liverpool and R. Golestanian, Phys. Rev. E 625488 (2000).

[7] R. Everaers, R. Bundschuh, and K. Kremer, Europhys. Lett., 29, 263 (1995); I.A. Nyrkova, A.N. Semenov and J-F. Joanny, J. Phys. (France) II, 6, 1411, (1996); S. Kumar and J. Singh, J. Stat. Phys., 89, 981 (1997); E.J. Janse van Rensburg et al, J. Stat. Phys., 85, 103 (1996).

[8] Z. Haijun, Y. Zhang, and Z.-C. Ou-Yang, Phys. Rev. Lett. 82, 4560 (1999).

[9] T.B. Liverpool, unpublished

[10] J.B. Lagowski, J. Noolandi, and B. Nickel, J. Chem. Phys., 95, 1266 (1991); A.M. Gupta, and S.F. Edwards, J. Chem. Phys., 98, 1588 (1993); B-Y Ha and D. Thirumalai, J. Chem. Phys., 103, 9408 (1995).

[11] J.F. Marko and E.D. Siggia, Macromolecules, 28, 8759 (1995); H. Flyvbjerg, cond-mat/0103417 D. Thirumalai and B-Y Ha, in Theoretical and Mathematical models in Polymer Research Ed. A. Grosberg, (Academic Press, New York 1998).

[12] F. David, and E. Guitter, Europhys. Lett., 5, 709 (1988); T.B. Liverpool, and S.F. Edwards, J. Chem. Phys., 103, 6716 (1995).

[13] T.B. Liverpool and A.C. Maggs, Macromolecules, 34, 6064 (2001); D.C. Morse, Phys. Rev. E 58, R1237-R1240 (1998).

[14] R.M. Hornreich, R. Liebmann, H.G. Schuster and W Selke, Z. Phys., B 35, 91 (1979).

[15] A. De Col and T.B. Liverpool, unpublished. 